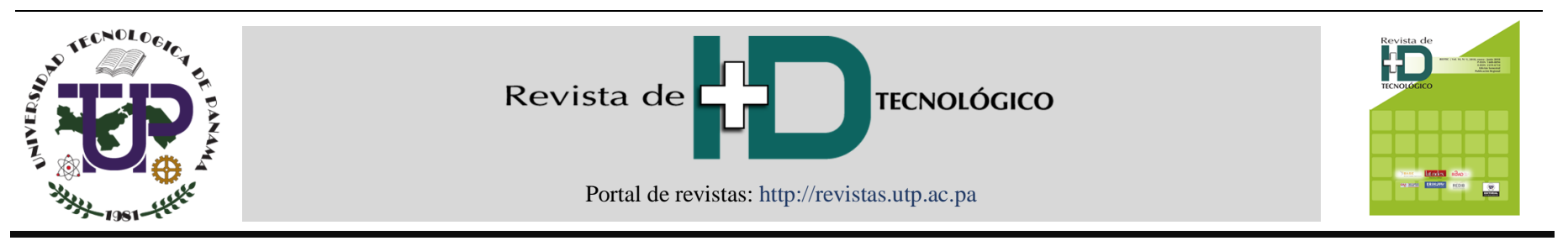

\title{
Los sistemas de interfaz cerebro-computadora basado en EEG: características y aplicaciones
}

\author{
Brain computer interface systems: characteristics and applications
}

\author{
I. Moreno ${ }^{1 *}$, E. Batista ${ }^{1}$, S. Serracín ${ }^{1}$, R. Moreno ${ }^{1}$, L. Gómez ${ }^{1}$, J. Serracín ${ }^{1}$, C. Boya ${ }^{2}$, J. Quintero ${ }^{1}$ \\ ${ }^{1}$ Grupo ROBOPROC, Centro Regional de Chiriquí, Universidad Tecnológica de Panamá, Panamá \\ *Autor de correspondencia: iveth.moreno@utp.ac.pa
}

\begin{abstract}
RESUMEN- Los sistemas de Interfaz Cerebro Computadora (BCI por su acrónimo en inglés) se basan en la característica del cerebro de emitir señales eléctricas, y cómo a través de la captación de estas señales, se pueden generar comandos de computador que controlen sistemas como sillas de ruedas con motores. Los sistemas BCI tienen diversas aplicaciones, pero su objetivo principal es brindar a personas con discapacidades motrices la posibilidad de transmisión de su intención a través de la modulación de las señales eléctricas en su actividad cerebral. En este artículo se presenta una breve descripción de lo que es una interfaz cerebro-computadora (BCI), sus características; y se describen sus orígenes y evolución, elementos constituyentes, tratamiento de señales cerebrales contemplando la función del cerebro, y las aplicaciones más relevantes.
\end{abstract}

Palabras clave-Cerebro, domótica, EEG, interfaz cerebro-computadora.

ABSTRACT - The Brain Computer Interface (BCI from its acronym in English) systems rely on the brain's characteristic of emitting electrical signals, and how through the register of these signals, computer commands controlling systems like wheelchairs with motors can be generated. BCI systems have many applications, but their principal objective relies on offering people with motor impairment the possibility to convey the intention through the electrical signal modulation from theirs brain activity. In this article it's presented a brief description about what is a Brain Computer Interface (BCI), its characteristics; and are described its origins, evolution, constituent elements, brain's signal treatment contemplating the cerebral function, and most relevant applications.

Keywords-Brain, domotics, EEG, brain computer interface.

\section{Introducción}

La Interfaz Cerebro Computadora (BCI por su acrónimo en inglés) es una interfaz asistida por computador que permite la interacción directa entre el cerebro y el entorno de un sujeto, a través de actuadores enlazados al computador. Este tipo de interfaz surge de la necesidad de establecer un nuevo canal de comunicación entre un sujeto y su entorno; que no dependa de sus vías nerviosas o musculares [1]. Estas interfaces se basan en la captación de señales asociadas a procesos mentales, aplicadas para la resolución de problemas de comunicación en pacientes, registrando señales neurofisiológicas adquiridas a través de EEG, MEG, fMRI, entre otras; facilitando con ello la interacción con el entorno a personas con discapacidades motrices severas [2].

Como seres vivos, las personas deben ser capaces de responder a los estímulos del ambiente para poder subsistir. En la vida diaria, esta respuesta se logra a través de dos vías esenciales: modificando el entorno en forma directa a través de los mecanismos musculares, o manifestando su pensamiento (comunicación), ya sea expresando sus sentimientos, deseos y/o ideas. No obstante, en algunos casos las funciones motrices se ven comprometidas debido a enfermedades como las lesiones medulares, infarto cerebral, esclerosis lateral amiotrófica, esclerosis múltiple, distrofia muscular, tumores o accidentes; por lo que sufren daños parciales o totales en subsistemas del cuerpo, lo que provoca perdida de la capacidad de respuesta natural (parcial o total) al entorno. Este tipo de discapacidad dio lugar al desarrollo de las tecnologías de interfaz cerebro computadora.

De forma inicial el primer sistema propiamente BCI fue desarrollado para contestar una pregunta: ¿Es posible utilizar señales eléctricas del cerebro para

Citación: I. Moreno, E. Batista, S. Serracín, R. Moreno, L. Gómez, J. Serracín, C. Boya y J. Quintero, "Los sistemas de interfaz cerebro-computadora basado en EEG: características y aplicaciones," Revista de I+D Tecnológico, vol. 15, no. 2, pp. (13-26), 2019.

Tipo de artículo: Original. Recibido: 3 de septiembre de 2018. Recibido con correcciones: 11 de abril de 2018. Aceptado: 26 de julio de 2019.

DOI. https://doi.org/10.33412/idt.v15.2.2230

Copyright: 2019 I. Moreno, E. Batista, S. Serracín, R. Moreno, L. Gómez, J. Serracín, C. Boya y J. Quintero. This is an open access article under the CC BY-NCSA 4.0 license (https://creativecommons.org/licenses/by-nc-sa/4.0/). 
comunicar información que permita controlar un dispositivo tal como una prótesis? Con tal interrogante en mente, el Dr. Jacques Vidal creó el primer sistema BCI, con el objetivo de evaluar si una persona se podía comunicar con una computadora, la cual interpretaría las señales del cerebro que posteriormente serían usadas para controlar una prótesis [3]. Desde Vidal, se han desarrollado varias versiones y mejoras en estos sistemas, con el propósito principal de recuperar o sustituir funciones motrices humanas; debido a vías neuromusculares inhabilitadas [4] [5] [6].

Un BCI es definido como "un sistema basado en una computadora que adquiere señales eléctricas del cerebro; las analiza y las traduce en comandos que son transmitidos a un dispositivo de salida para llevar a cabo una acción deseada", según [7]. La idea central es capturar la intención del usuario implícita en las señales eléctricas de su cerebro, procesando dichas señales, y extrayendo sus características y las relaciones entre las mismas, para una posterior interpretación a comandos (i.e. órdenes), que son ejecutadas por una computadora $\mathrm{u}$ otro dispositivo a través de actuadores. En la actualidad los sistemas BCI se consideran una herramienta de gran potencial como medio de comunicación alternativo, actuador, y medio de restablecimiento y rehabilitación de funciones motrices en sujetos con discapacidades neuromotoras [8].

La interfaz cerebro computador por la naturaleza de su método de adquisición de señales puede ser de tipo invasiva: mediante electrodos implantados en la superficie de la corteza cerebral o en su interior; o no invasiva: mediante electrodos ubicados en la superficie del cuero cabelludo. Tras esta adquisición de señales, la BCI se convierte en un nuevo canal de salida del cerebro que, como otros canales, puede regular su respuesta para adaptarla al objetivo propuesto [9]; es decir, el cerebro a través de una imaginación motora por ejemplo, emite una señal que la BCI transforma en una orden para controlar acciones de su entorno.

En este artículo se presenta brevemente lo que son los sistemas BCI, enfocados principalmente a sistemas no invasivos, sus características y aplicaciones más relevantes. El desarrollo del documento se realiza de la siguiente manera: en la sección dos, el cerebro modulación eléctrica (i.e. aspectos de la actividad eléctrica cerebral); en la sección tres, métodos de adquisición de señales; en la sección cuatro componentes (i.e. etapas) de un sistema BCI; en la sección cinco, tipos de señales utilizadas para control BCI; en la sección seis, aplicaciones; en la sección siete se hablan de estudios actuales sobre la retroalimentación; en la sección ocho, sobre "BCI inteligente"; y, finalmente la discusión.

\section{EI Cerebro: Modulación Eléctrica}

El cerebro constituye el núcleo del Sistema Nervioso Central (CNS), y su función es el procesamiento de información (i.e. estímulos) recibida desde los sentidos con el objetivo de preparar y ejecutar una respuesta [10]. Entre los tipos de respuesta cerebral, está la actividad electrofisiológica. La existencia de esta naturaleza en las respuestas cerebrales brinda la posibilidad de rastrear procesos mentales reflejados en las señales eléctricas.

\subsection{Explorando el cerebro.}

A finales del siglo XVII el estudio enfocado al cerebro a manos del Dr. Gustav Fritsch dio un aporte importante para conformar la neurociencia moderna, a través de la comprobación de la naturaleza eléctrica en el funcionamiento del cerebro y la existencia de la corteza motora. La comprobación se consiguió al estudiar los cerebros de pacientes provenientes de la guerra entre Prusia y Dinamarca [11].

Se reconoció que la actividad cerebral se debe al funcionamiento de células especializadas (i.e. nerviosas) llamadas neuronas, las cuales poseen propiedades eléctricas y químicas; siendo las neuronas la fuente de la naturaleza eléctrica que posee el cerebro [12].

\subsection{Las Neuronas}

Las neuronas existen en grandes cantidades; con variable morfología; sus componentes principales son las dendritas, el soma y el axón. Las dendritas reciben la información y se conectan con axones; mientras que el axón proyecta información y se conecta a dendritas; a estas conexiones se les denomina sinapsis [13]. Estas partes, en conjunto con el núcleo, constituyen el cuerpo de la neurona [14].

Las neuronas transmiten la información mediante respuestas químicas y eléctricas. En forma predominante, entre neuronas se comunican entre sî mediante respuestas químicas utilizando neurotransmisores [14], los neurotransmisores desencadenan cambios de potencial eléctrico dentro del cuerpo de la neurona denominados potenciales de 
acción [15]; este tipo de actividad eléctrica es similar a la de un circuito eléctrico [16].

Esencialmente, las neuronas funcionan como pequeñas unidades de proceso y/o transmisión de información, que constituyen redes de procesamiento para operaciones más complejas, a través de su configuración en arreglos de diferentes tipos de neuronas. Estos arreglos conforman estructuras con diferentes especializaciones.

\subsection{Divisiones funcionales del cerebro}

De forma global, las estructuras conformadas por neuronas en el cerebro pueden percibirse como tres grandes estructuras cerebrales: la corteza cerebral (i.e. neocorteza), el cerebelo y el sistema límbico.

La corteza cerebral se encarga de funciones superiores y del control de los actos voluntarios [17] y se puede dividir en cuatro partes llamadas lóbulos. Estos lóbulos son: frontal, parietal, temporal y occipital; y exceptuando al frontal estos se encargan de procesar la información adquirida a través de los sentidos.

El cerebelo procesa los impulsos eléctricos provenientes de los sistemas esquelético y muscular; incluyendo los sentidos y realiza funciones de coordinación (avanzadas), organización (de ejecución), optimización de adquisición sensorial de información, y ajuste y recalibración de sistemas [18] [19] [20] [21] [22]. Finalmente, el sistema límbico es el encargado de procesar emociones [23]; además, en este sistema tiene lugar la memoria a largo plazo.

La neocorteza gobierna la creatividad, inteligencias, emociones, comportamiento y la memoria [24] además de la ejecución. Al tener múltiples estructuras con funciones diferenciadas, puede llevar a cabo acciones simultáneas de diferente naturaleza, como los pensamientos, habla, y/o coordinación (básica) y ejecución motora (e.g. como caminar); siempre y cuando no se padezca de alguna limitante.

\section{Métodos de adquisición de señales}

\subsection{Antecedentes de la EEG}

Entre los primeros científicos que hicieron aportes en el campo de la neurofisiología, más concretamente en el estudio de la electrogénesis cerebral, se destacan: Richard Caton, que en 1875 realizo pruebas en perros y logro confirmar actividad eléctrica en la "sustancia gris" [25], en el 1891 Adolf Beck hace las primeras observaciones sobre potenciales evocados visuales y es el primero en plantear la existencia de actividad eléctrica no sólo en el cerebro sino también a lo largo de la médula espinal y su posible variación dependiendo de la actividad realizada [26]; pero fue el alemán Hans Berger el inventor del término "electroencefalograma" y el primero en investigar y comprobar la existencia de señales eléctricas en el cerebro humano, por lo que se le considera el padre de la electroencefalografía (EEG) [27].

El concepto de electroencefalografía (EEG) hace referencia a una técnica de adquisición de la señal eléctrica cerebral de forma no invasiva; en la que se registra la región emisora, la polaridad y los cambios espacio temporales de dichas señales por medio de electrodos superficiales y basales que transforman las corrientes iónicas del tejido cerebral en corrientes eléctricas en la superficie craneal.

Es Berger quien en 1924 utilizando un galvanómetro de cuerda logra registrar los primeros "ritmos" en su hijo [28], es decir, es el primero en reconocer los patrones característicos de la señal eléctrica que se observan en la presencia de eventos o comportamientos específicos del individuo. Para esta recopilación de señales utilizó electrodos de aguja de distintos materiales (zinc, platino, plomo, entre otros) en el cuero cabelludo de forma invasiva, pero sin llegar a penetrar el cráneo, que era la única técnica utilizada en la época. El proceso finalizaba con la impresión de las señales por medio del reflejo de un espejo y papel de bromuro de plata que se movía a una velocidad de $3 \mathrm{~cm} / \mathrm{s}$ [29].

No es hasta 1930 cuando hace una recopilación de más de 1100 registros de 76 individuos, donde clasifica dos tipos de ritmos según el voltaje y frecuencia de sus respectivas ondas; a las de mayor voltaje y menor frecuencia las llamó alfa $(\alpha)$ y a las de menor voltaje y mayor frecuencia beta $(\beta)[30]$.

Debido a su falta de conocimiento técnico y la escasa disponibilidad del equipo apropiado para tomar las mediciones, no pudo avanzar mucho más en sus investigaciones. Pero en 1934 Adrian y Matthews corroboran el trabajo de Berger al utilizar amplificadores y galvanómetros más sofisticados y potentes [31].

\subsection{EEG en la actualidad}

En la actualidad con los avances en la electrónica tanto el proceso de adquisición como el de análisis de las señales se han optimizado considerablemente. 
Permitiendo a su vez que los métodos de colocación de los electrodos sean menos invasivos, evitando así mayores riesgos para los pacientes.

Todo el sistema nervioso puede generar electricidad, unas zonas con mayor intensidad que otras. Para propósitos de obtención de señales EEG, se toman en cuenta las células piramidales de las capas III y IV de la neocorteza del cerebro [31]; las señales generadas son superposiciones de todos los potenciales de las neuronas perpendiculares a la ubicación del electrodo [32].

Los principales tipos de electrodos superficiales se pueden clasificar según la forma en que se consigue la señal en:

- Electrodos de aguja: de uso poco común debido a su complejidad. Se suele restringir su uso a neonatos y cuidados intensivos [33].

- Electrodos adheridos: pequeños discos metálicos que se caracterizan por tener muy baja resistencia de entre 1 a $2 \mathrm{~K} \Omega$. A su vez estos se pueden subdividir en:

- Húmedos: los que por medio de una pasta o solución conductora facilitan el intercambio de información, aptos para periodos de registro cortos [25], [31].

- Activos-secos: se utilizan materiales que tengan estabilidad química, resistencia mecánica y buenas propiedades eléctricas; es apto para periodos de registro prolongados [25].

- Electrodos de casco o malla: se utiliza un casco elástico con ranuras para los electrodos, variando el tamaño del casco según el paciente [31].

Por otro lado, los electrodos basales o también llamados, especiales, utilizan otras vías de acceso a la base del cerebro, como lo son la faríngea y la timpánica [25].

\subsection{Disposición de los electrodos}

Desde 1958 la Federación Internacional de Sociedades de Electroencefalografía y Neurofisiología Clínica estableció la norma estándar de colocación de electrodos. La norma 10-20, que divide las distancias del cráneo en distancias que corresponden al 10\% la longitud de arco entre el inion y el nasion, con una trayectoria a través del vertex; el $20 \%$ se refiere a la distancia entre dos electrodos, como una separación equidistante. En esta convención cada punto posee una letra y un subíndice; las letras hacen referencia al lóbulo cerebral al que corresponden y los subíndices "z" para la línea media del cráneo indicando "cero", mientras que los subíndices numéricos pares indican el hemisferio derecho y los impares el hemisferio izquierdo. De esta forma se puede decir que: $F$ corresponde al lóbulo frontal, $\mathrm{T}$ al temporal, $\mathrm{O}$ al occipital y $\mathrm{P}$ al parietal; $\mathrm{C}$ corresponde al vertex y $\mathrm{A}$, a la zona del pabellón auditivo [24].

Se pueden distinguir dos arreglos principales (ver Figura 5) para el análisis de las señales EEG:

- Monopolar o referencial: este arreglo utiliza un "electrodo activo" (i.e. cambiante) en conjunto con un electrodo a modo de referencia (tierra), que suelen colocarse en el lóbulo de la oreja o en la barbilla; este principio se aplica a los demás electrodos de forma individual según el número de canales disponibles.

- Bipolar o diferencial: éste toma los valores monopolares de los electrodos y realiza una sustracción entre los mismos generando un canal. Este arreglo disminuye la contaminación de la señal captada para el análisis.

Cabe destacar que todos los potenciales son explícitamente bipolares, no obstante, el término es utilizado porque se entiende que el valor de un voltaje a electrodo activo (i.e. la medida monopolar) es necesariamente ya referenciado a un valor tierra, por lo que es estrictamente bipolar [34].

\section{Funcionamiento de un sistema BCI}

Un sistema BCI detecta la actividad eléctrica generada en el cerebro. Esta actividad eléctrica produce señales que pueden contener información acerca de una tarea mental específica, sea consciente (e.g. Ritmos sensoriomotores) o inconsciente (e.g. modulaciones P300); a dicha señal se le extraen sus características más relevantes de tal forma que a partir de estas, se pueda identificar si se trata de uno u otro proceso mental [35], Esto es, que de cada proceso o tarea mental se observará un registro EEG diferente. A la persona se le puede solicitar que realice estas tareas de manera repetitiva para obtener un promedio de cada tipo de señal.

Las características que se extraen a estas dos señales deben ser óptimas para identificación, es decir, las que permitan de manera más eficiente diferenciarlas una de la otra. Estas características pueden ser la cantidad de energía en bandas específicas de frecuencia, 


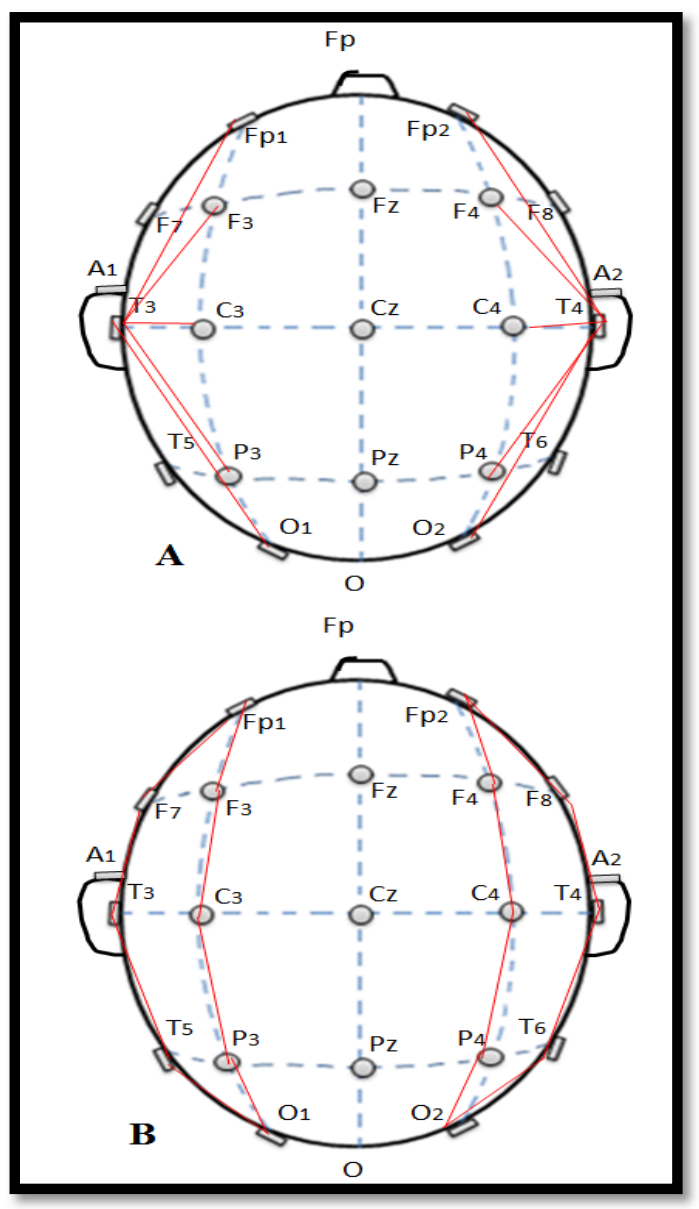

Figura 1. Diagrama de arreglo referencial (A) y diferencial (B).

su amplitud, su duración, su distribución espacial, o relaciones entre las características anteriormente mencionadas, entre otras [35]. Posteriormente, los valores de estas características asociados a cada tarea mental pueden ser utilizadas como comandos a través de un actuador acoplado a la interfaz. Como ejemplo la imaginación del movimiento del brazo izquierdo puede ser el comando "encender la luz" (a través de un interruptor) y la señal generada por la imaginación del movimiento de la pierna derecha para "apagar la luz" (a través del interruptor). De esta manera, si la persona desea encender la luz sólo tiene que imaginar mover el brazo izquierdo y para apagarla, imaginar el movimiento de la pierna derecha.

Un sistema de BCI detecta y procesa las señales, compara las características de la señal obtenida del sujeto con las de un patrón en una base de datos, identifica la señal en base a sus características y la computadora selecciona el comando asociado a tal patrón. El comando se utiliza para controlar un dispositivo externo (acoplado a la interfaz) tal como un interruptor de luminaria o el motor de una silla de rueda [36]. La materialización del deseo del usuario y la consecuente conciencia de ello, cierra un ciclo de control que se inicia en el pensamiento ( $u$ otros procesos mentales).

Aunque en estos ejemplos se obvian algunas complejidades que surgen de una BCI, ilustra su fin, el cual es crear un nuevo canal para comunicar las intenciones o deseos de una persona.

Para la comprensión del proceso de un BCI en forma más detallada, el sistema se divide en cuatro partes [37]: Adquisición de señales, extracción de características, asignación de comandos y salida o acción del comando. Estas partes son controladas por un protocolo que define el inicio y tiempo de cada operación, además de los detalles del procesamiento de las señales.

\subsection{Adquisición de señales}

Una vez adquirida la señal EEG, siguiendo alguno de los arreglos estándar (Figura 1), a través de un sistema no invasivo, se amplifica a un nivel adecuado para su procesamiento. En este punto puede aplicarse algún tipo de filtrado analógico para remover señales indeseables tales como la señal de 50/60 Hz de la línea de distribución eléctrica y/o artefactos de baja frecuencia; cabe destacar que el uso de filtros analógicos puede generar derivas de baja frecuencia en las señales. Finalmente, las señales se digitalizan, se agrupan en bloques de muestras y se transmiten en secuencia a una computadora para su almacenamiento y procesado.

\subsection{Extracción de características}

Este proceso analiza las señales con el objetivo de obtener características relevantes, de tal manera que puedan ser correlacionadas con la intención del usuario.

Los electrodos de un EEG registran las variaciones de voltaje del cuero cabelludo en un momento dado. No obstante, estas variaciones no representan explícitamente ninguna intención por parte del usuario. Por ello, se recurre al reconocimiento de patrones en las señales, o más propiamente, a los patrones entre las características de las señales. Para este efecto las señales se acondicionan, posteriormente se capturan las características y finalmente se acondicionan las 
características. En el acondicionamiento de la señal, se realiza filtrado digital en la frecuencia y en el espacio.

Los filtros en la frecuencia remueven derivas de baja frecuencia introducidas por el uso de filtros analógicos (en caso de haberse utilizado) y se remueven rangos de frecuencia irrelevantes para el análisis de la señal. También pueden utilizarse filtros pasa banda y de muesca digitales, para remover interferencia de la línea y artefactos en caso de no haberse filtrado analógicamente.

Los filtros en el espacio buscan el filtrado de los canales (i.e. diferencia de potencial entre electrodos), de forma que se enfatice en aspectos relevantes de la señal. El filtro espacial, es una matriz de pesos (i.e. parámetros) $\mathrm{W}$, que multiplicada por el arreglo de señales $X$ que ingresa de los canales, produce un set de canales $\mathrm{Y}$ con señales filtradas, es decir:

$$
Y=W X
$$

Que es lo mismo que:

$\left[\begin{array}{cccc}y_{11} & y_{12} & \cdots & Y_{1 P} \\ y_{21} & y_{22} & & \\ & \vdots & \ddots & \vdots \\ y_{M 1} & & \cdots & Y_{M P}\end{array}\right]=\left[\begin{array}{cccc}w_{11} & w_{12} & \cdots & w_{1 N} \\ w_{21} & w_{22} & & \\ & \vdots & \ddots & \vdots \\ w_{M 1} & & \cdots & w_{M N}\end{array}\right]\left[\begin{array}{cccc}x_{11} & x_{12} & \cdots & x_{1 P} \\ x_{21} & x_{22} & & \\ & \vdots & \ddots & \vdots \\ x_{N 1} & & \cdots & x_{N P}\end{array}\right]$

En esta expresión: $\mathrm{P}$ indica el número de muestra de señal digital sucesiva para cada un canal N; las filas $\mathrm{W}$ representan $\mathrm{N}$ pesos de muestras para esos canales. [35]. Esta matriz de peso $\mathrm{W}$ puede crearse mediante diferentes técnicas, que pueden ser dependientes o independientes de la data de alguna actividad cerebral particular.

Los filtros independientes de la data se basan en ajustes de relaciones geométricas. Estos pueden ser:

- Referencia común promedio (CAR): promedia el potencial de todos los electrodos y usa el valor resultante como una referencia para obtener el potencial en un electrodo dado. Esto es:

$$
\boldsymbol{V}_{\boldsymbol{n}}=\Phi\left(r_{n}\right)-\frac{1}{N} \sum_{n=1}^{\infty} \Phi\left(r_{n}\right)
$$

$\boldsymbol{n}$ : se refiere al número de electrodo; $\Phi\left(r_{\boldsymbol{n}}\right)$ : se refiere al potencial dipolar del electrodo $n$ de interés respecto al infinito; la sumatoria promediada representa la referencia común; y $\boldsymbol{V}_{\boldsymbol{n}}$ es el voltaje ajustado de un electrodo $\boldsymbol{n}$.

- Laplaciano: segundo derivativo espacial de potenciales en el cuero cabelludo. Para un electrodo dado elimina los potenciales que son comunes a los electrodos circundantes. Se puede realizar hasta con un mínimo de 5 electrodos (i.e. 4 ortogonales al electrodo de interés), lo que se conoce como Laplaciano del vecino más cercano [38], y se expresa como:

$$
\boldsymbol{V}_{\boldsymbol{n}}=\Phi\left(r_{\boldsymbol{n}}\right)-\frac{1}{4} \sum_{i \in \Theta} \Phi\left(r_{\boldsymbol{n}}\right)
$$

Donde $\boldsymbol{V}_{\boldsymbol{n}}$ : es el voltaje ajustado; $\Phi\left(r_{\boldsymbol{n}}\right)$ : al potencial dipolar del electrodo $\boldsymbol{n}$ de interés respecto al infinito; y $\Theta$ : representa los índices de potencial de los electrodos circundantes.

Los filtros dependientes de la data ajustan el filtro en base a información (previamente obtenida) asociada a una tarea cerebral dada. Entre estos, se tiene:

- Análisis de Componentes Principales (PCA): Realiza un ajuste dimensional a la data, para obtener varianza máxima y ortogonalidad en las componentes principales; reubicando el eje en el centroide de datos (de cada canal), por medio de la sustracción del promedio de $\mathrm{P}$ muestras para cada muestra j de un canal i de la matriz X, es decir:

$$
x_{i j}-\frac{1}{P} \sum_{j=1}^{P}\left(x_{i j}\right)=x_{i j}-\bar{x}_{\imath}=x_{i j}{ }^{\prime}
$$

Para generar una matriz ajustada $X^{\prime}$ :

$$
X^{\prime}=\left[\begin{array}{cccc}
x_{11}{ }^{\prime} & x_{12}{ }^{\prime} & \cdots & x_{1 P}{ }^{\prime} \\
x_{21}{ }^{\prime} & x_{22}{ }^{\prime} & & \\
\vdots & \ddots & \vdots \\
x_{N 1}{ }^{\prime} & & \cdots & x_{N P}{ }^{\prime}
\end{array}\right]
$$

De forma que se obtenga la matriz de covarianzas de esta matriz $X^{\prime}$, y así se calculen los autovalores y auto-vectores de dicha matriz de covarianza. Cada conjunto de auto-vectores correspondiente a un autovalor de $X^{\prime}$, constituye los pesos (i.e. parámetros) de una fila de la matriz $\mathrm{W}$, representando el conjunto de canales filtrados $\mathrm{Y}$ mediante:

$$
Y=W X^{\prime}
$$

Esta técnica es apropiada cuando los canales están muy correlacionados y/o son mayores en número a las fuentes independientes.

- Patrones Espaciales Comunes (CSP): 
El principio es el mismo del PCA, pero considera condiciones específicas de operación. Realiza una configuración dual de ejes ortogonales, de forma que se produzca máxima varianza para una condición dada y mínima para otra. Esto los hace muy viables en una discriminación dual.

- Análisis de Componentes Independientes (ICA): Es una técnica similar a PCA, pero que además busca que las componentes sean estadísticamente independientes.

Estas mismas técnicas de análisis dependiente de la data, pueden utilizarse para identificar artefactos $\mathrm{y}$ removerlos.

Posteriormente, se realiza la captura (i.e. extracción propiamente) de las características de interés en la señal, utilizando una técnica que sea apropiada para ello, y así conformar vectores de características. Para este efecto, se utilizan técnicas (i.e. modelos o algoritmos) como:

- Peak Picking: captura máximos y/o mínimos para un bloque de y/o su tiempo de ocurrencia como característica.

- Integración de bloque: realiza un promedio de un bloque y toma este valor como característica.

- Bandas de energía (mediante filtro): se toman valores absolutos o al cuadrado de amplitudes de la señal a frecuencias específicas.

- Transformada Rápida de Fourier (FFT): captura características espectrales (e.g. magnitud y fase) mediante transformada de Fourier:

$$
\begin{gathered}
X(\omega)=\int_{-\infty}^{\infty} x(t) \cdot e^{-j \omega t} d t \\
X(\omega)=a(\omega)+j b(\omega)
\end{gathered}
$$

Con magnitud $|X(\omega)|$ y fase $\theta$ denotadas como:

$$
\begin{array}{r}
|X(\omega)|=\sqrt{a^{2}(\omega)+b^{2}(\omega)} \\
\theta=\arg (X(\omega))=\tan ^{-1}\left(\frac{b(\omega)}{a(\omega)}\right)
\end{array}
$$

Para cada frecuencia $\omega$, por medio de un algoritmo eficiente para obtener la Transformada de Fourier discreta.
- Transformada Wavelet: captura características similares a las de Fourier utilizando ondas madres (i.e. modulaciones de ondas finitas) en vez de senos y cosenos (i.e. ondas infinitas), permitiendo crear ventanas tiempo-frecuencia.

Además de otras técnicas como Modelado AutoRegresivo (A-R), Dimensión Fractal, Parámetros Hjorth, Phase locking value (PLV), Coherencia, Distancia de Mahalanobis, entre otros [39].

Cabe destacar, que la técnica utilizada debe ser congruente con el tipo de características que se esté extrayendo. Adicionalmente, se debe tener cuidado con la selección de características. Las características deben cumplir con tres requisitos: ser comunes a un usuario o población, ser relativamente estacionarias y rastreables, y su modulación debe ser a voluntad (del usuario) o por sus procesos de atención; de lo contrario, no serán efectivas en aplicaciones BCI.

Las características extraídas se acondicionan: realizando normalización para expresar las características en términos de valores unitarios o logarítmicos, y finalmente son utilizados para conformar vectores de características que se traducen posteriormente en la siguiente etapa.

\subsection{Interpretación de comandos}

Las características de las señales se transfieren como vectores a un algoritmo basado en un modelo matemático para la interpretación de comandos. El cual convierte las características en comandos apropiados para la salida del sistema BCI. Esto se realiza utilizando modelos matemáticos que puedan diferenciar grupos de características a través de zonas en los espacios entre ellas, de tal forma que la posición de una respecto a la tendencia del modelo matemático, active una señal que desencadene un comando. Entre estos modelos matemáticos se encuentran diferentes tipos de discriminantes como Análisis Discriminante Lineal (LDA), método Robusto para Análisis Discriminante Lineal (RLDA), Análisis Discriminante Cuadrático (QDA), Máquinas de Soporte Vectorial (SVM), Bagging, Random Forest, Boosting, Learning Vector Quantization, que son viables como diferenciadores discretos; y funciones de regresión que es más adecuada para generación de comandos continuos. Además de estas aproximaciones se encuentran las redes neuronales, y las redes con back propagation que 
pueden desempeñar las funciones del algoritmo de extracción de características y traducción al mismo tiempo [39].

La generalización del algoritmo será mayor para una mayor cantidad de datos al parametrizar el modelo, y será menor mientras más parámetros sean usados en el modelo [40]. Además, se debe tomar en cuenta que el algoritmo de asignación de comandos debe ser dinámico de tal forma que se adapte a cambios espontáneos en las características de las señales, sea por condicionamiento operante (i.e. ajuste realizado por el usuario) [41], por aprendizaje de máquina [42] o por ambas simultáneamente [43]; y debe asegurar que el usuario tenga la posibilidad de un eficiente rango de valores para controlar un dispositivo externo.

\subsection{Salida del sistema BCI}

Los comandos desde el algoritmo de interpretación operan sobre dispositivos externos, tales como la selección de una letra, control del cursor, brazo robótico, silla de ruedas, luces etc. La operación del dispositivo de salida provee la realimentación al usuario, y de esta forma se cierra el ciclo de control.

\section{Señales inherentes a los eventos cerebrales utilizadas en un BCI}

Las señales que se registran como actividad EEG son cambios en los potenciales eléctricos en zonas del cuero cabelludo como se mencionó previamente. Estos potenciales se encuentran distribuidos a lo largo de todo el cuero cabelludo. No obstante, como el cerebro es un sistema de redes complejo que realiza distintos tipos de procesamiento dependiendo de la zona funcional, se pueden encontrar tipos de señales muy diferenciados dependiendo de su región de generación, asociadas a procesos mentales de diversa índole. Algunas señales se modulan a través de procesos metabólicos, otras dependen de niveles motivacionales y de atención del usuario; y, por último, se encuentran señales que se pueden modular a voluntad.

Si bien existe gran variedad de señales, para una aplicación práctica de BCI mediante un sistema EEG, se pueden utilizar [41-52]:

- P300: componente endógena (i.e. tardía) de un potencial relacionado a eventos (ERP), que se asocia con procesamiento de ciertos eventos y ocurre aproximadamente a $300 \mathrm{~ms}$.
- Potenciales evocados de estado estable (SSEP): son potenciales evocados de estado estable generados a partir de estímulos a una frecuencia determinada, que producen una respuesta a una frecuencia aproximada a la del estímulo. Entre estos se tienen:

- SSVEP (Steady State Visual Evoked Potentials): potencial generado por estímulos visuales.

- SSSEP (Steady State Somatosensory Evoked Potentials): potencial evocado generado por estímulos somatosensoriales.

- ASSEP (Auditory Steady State Evoked Potentials): potencial evocado generado por estímulos auditivos.

- Potenciales Corticales Lentos (SCP): Son potenciales asociados a actividades motrices o de imaginación motriz, y entre estos se tienen:

- SCP BP (Slow Cortical Potentials Bereitschaftspotentials): Es un potencial cortical asociado a preparación para actividad motriz.

- SCP CNV (Slow Cortical Potentials Contingent Negative Variation): Es un potencial cortical asociado a la finalización de una actividad motriz.

- Ritmos sensoriomotores (SMR): son disminuciones y/o aumentos en las bandas de frecuencia Mu1 de $8-10 \mathrm{~Hz}, \mathrm{Mu} 2$ de $10-13 \mathrm{~Hz}$ y Beta de 18-25 Hz [56] en el cuero cabelludo, asociadas a actividad o imaginación motora.

A continuación, la tabla 1 detalla aspectos asociados a cada una de estas señales.

\section{Aplicaciones}

La aplicación del BCI se refiere a los propósitos o dispositivos específicos a los que se aplican los comandos de salida de un sistema BCI [57]. Diversos artículos se han encargado de recopilar las aplicaciones más importantes. Mark enfoca su investigación en aplicaciones clínicas, y divide estas en dos grandes grupos: tecnologías de asistencia y neuro-rehabilitación. Dentro de las tecnologías de asistencia se tienen las áreas de comunicación, control de movimiento, control del ambiente y locomoción; similar clasificación es presentada por Moore [58] en su artículo sobre las aplicaciones en el mundo real de esta tecnología. 
Tabla 1. Tipos de Señales utilizados en aplicaciones BCI

\begin{tabular}{|c|c|c|c|c|c|c|c|}
\hline Tipo de & P300 & SSVEP & SSSEP & ASSEP & SCP BP & SCP CNV & SMR \\
\hline $\begin{array}{l}\text { Apariencia de la } \\
\text { señal }\end{array}$ & Deflexión positiva & $\begin{array}{l}\text { Serie de deflexiones } \\
\text { positivas y negativas }\end{array}$ & $\begin{array}{l}\text { Serie de deflexiones } \\
\text { positivas y negativas }\end{array}$ & $\begin{array}{l}\text { Serie de deflexiones } \\
\text { positivas y negativas }\end{array}$ & Polaridad negativa & Polaridad negativa & $\begin{array}{l}\text { Aumentos } \\
\text { disminuciones }\end{array}$ \\
\hline $\begin{array}{l}\text { Condiciones } \\
\text { generación }\end{array}$ & $\begin{array}{ll}\text { Estímulo } & \text { entregado } \\
\text { bajo } & \text { paradigma } \\
\text { excéntrico } & \end{array}$ & $\begin{array}{l}\text { Exposición a estímulo } \\
\text { visual de frecuencia } \\
\text { constante }\end{array}$ & $\begin{array}{l}\text { Exposición a estímulo } \\
\text { táctil a frecuencia } \\
\text { constante }\end{array}$ & $\begin{array}{l}\text { Exposición a estímulo } \\
\text { auditivo a frecuencia } \\
\text { constante }\end{array}$ & $\begin{array}{l}\text { Intención de iniciación } \\
\text { de movimientos }\end{array}$ & $\begin{array}{l}\text { Incidencia de estímulo } \\
\text { que requiera respuesta. }\end{array}$ & $\begin{array}{l}\text { Intención de iniciación } \\
\text { de movimientos }\end{array}$ \\
\hline Dominio de análisis & Temporal & Frecuencia y/o tiempo & Frecuencia & Frecuencia & Temporal & Temporal & Frecuencia \\
\hline $\begin{array}{l}\text { Deflexiones } \\
\text { alteraciones } \\
\text { importantes }\end{array}$ & $\begin{array}{l}\text { Deflexiones } \\
\text { P300: Positiva a } 300 \\
\text { ms }\end{array}$ & $\begin{array}{l}\text { Deflexiones } \\
\text { N70: Negativa a } 70 \mathrm{~ms} \\
\text { P100: Positiva a } 100 \\
\text { ms }\end{array}$ & $\begin{array}{l}\text { Picos de frecuencias } \\
\text { mayores con estímulos } \\
\text { en las manos entre los } \\
21 \text { a } 26 \mathrm{~Hz} \text {. }\end{array}$ & $\begin{array}{l}\text { La magnitud del } \\
\text { segundo armónico es } \\
\text { mayor para } \\
\text { frecuencias entre } 5 \text { y } \\
20 \mathrm{~Hz} \text {. }\end{array}$ & $\begin{array}{l}\text { Ocurren de } 500 \text { a } 1000 \\
\text { ms, antes de un } \\
\text { movimiento iniciado a } \\
\text { voluntad. }\end{array}$ & $\begin{array}{l}\text { Ocurren de } 200 \text { a } 500 \\
\text { ms posterior a un } \\
\text { estímulo (que requiera } \\
\text { acción) en S1. }\end{array}$ & $\begin{array}{l}\text { Ocurren entre los } 8- \\
12 \mathrm{~Hz}, 18-30 \mathrm{~Hz} \text { y } \\
\text { de } 30-200 \mathrm{~Hz} \text {. }\end{array}$ \\
\hline Amplitud & $\begin{array}{l}10 \text { a } 20 \mathrm{uV} \\
\text { Percibido como: } \\
4 \text { a } 10 \mathrm{uV}\end{array}$ & $\begin{array}{l}\text { Amplitudes en } \\
\text { armónicos } \\
\text { decrecientes a medida } \\
\text { aumenta el armónico }\end{array}$ & $\begin{array}{l}\text { Primer armónico de } \\
\text { modulación altamente } \\
\text { dominante. }\end{array}$ & $\begin{array}{l}\text { Diferentes frecuencias } \\
\text { tonales proporcionan } \\
\text { funciones con } \\
\text { amplitudes similares. }\end{array}$ & $\begin{array}{l}\text { Varía dependiendo del } \\
\text { tipo de movimiento, } \\
\text { músculo involucrado y } \\
\text { estado psicológico. }\end{array}$ & $\begin{array}{l}\text { Varía dependiendo de } \\
\text { la tarea específica y es } \\
\text { afectada por factores } \\
\text { motivacionales. }\end{array}$ & $\begin{array}{l}\text { Varía dependiendo del } \\
\text { músculo de interés del } \\
\text { usuario y de la zona de } \\
\text { generación. }\end{array}$ \\
\hline $\begin{array}{l}\text { Características al } \\
\text { modularse }\end{array}$ & $\begin{array}{l}\text { Amplitud inversamente } \\
\text { proporcional a ra } \\
\text { Probabilidad } \\
\text { estímulo. } \\
\text { Mayores dificultad de } \\
\text { tarea o mayores } \\
\text { tiempos entre estímulo, } \\
\text { implican mayores } \\
\text { amplitudes. }\end{array}$ & $\begin{array}{l}\text { Frecuencia similar a la } \\
\text { del estímulo. }\end{array}$ & $\begin{array}{l}\text { Frecuencia similar a la } \\
\text { del estímulo. } \\
\text { Amplitudes mayores } \\
\text { en zonas } \\
\text { contralaterales a la } \\
\text { región del cerebro } \\
\text { asociada al proceso. }\end{array}$ & $\begin{array}{l}\text { Frecuencia similar a la } \\
\text { del estímulo. } \\
\text { Decremento lineal en } \\
\text { amplitud y } \\
\text { lineal en } \\
\text { asociadonento } \\
\text { decremento } \\
\text { intensidad } \\
\text { estímulo. }\end{array}$ & $\begin{array}{l}\text { Picos de mayor de } \\
\text { amplitud en la corteza } \\
\text { se muestran contra } \\
\text { lateralmente a la zona } \\
\text { asociada al proceso } \\
\text { requerido. }\end{array}$ & $\begin{array}{l}\text { La amplitud cambia } \\
\text { con } \\
\text { entrenamiento/uso de } \\
\text { este sistema. }\end{array}$ & $\begin{array}{l}\text { Disminuye el nivel de } \\
\text { energía entre las } \\
\text { frecuencias } 8-10 \mathrm{~Hz} \text {, } \\
\text { en las regiones } \\
\text { contralaterales del } \\
\text { cerebro, a la } \\
\text { imaginación motora. }\end{array}$ \\
\hline $\begin{array}{l}\text { Región de modulación } \\
\text { accesible mediante } \\
\text { EEG. }\end{array}$ & $\begin{array}{l}\text { Lóbulo Parietal y } \\
\text { Occipital }\end{array}$ & $\begin{array}{l}\text { Áreas occipitales, } \\
\text { especialmente corteza } \\
\text { visual primaria. }\end{array}$ & S1 & $\begin{array}{l}\text { S1 y regiones de } \\
\text { procesamiento } \\
\text { auditivo. }\end{array}$ & PMC, SMA, M1 y S1 & $\begin{array}{l}\text { SMA, S1, S2, Áreas } \\
\text { Frontales y/o M1. }\end{array}$ & $\begin{array}{l}\text { PM, SMA, S1, } \\
\text { especialmente M1. }\end{array}$ \\
\hline $\begin{array}{l}\text { Naturaleza } \\
\text { modulación }\end{array}$ & $\begin{array}{l}\text { Inconsciente: Función } \\
\text { de reconocimiento }\end{array}$ & $\begin{array}{l}\text { Inconsciente: } \\
\text { Función de } \\
\text { procesamiento visual }\end{array}$ & $\begin{array}{l}\text { Inconsciente Función } \\
\text { de procesamiento } \\
\text { táctil }\end{array}$ & $\begin{array}{l}\text { Inconsciente Función } \\
\text { de procesamiento } \\
\text { auditivo }\end{array}$ & $\begin{array}{l}\text { Consciente } \\
\text { Función asociada a } \\
\text { operaciones motrices }\end{array}$ & $\begin{array}{l}\text { Consciente } \\
\text { Función asociada a } \\
\text { operaciones motrices }\end{array}$ & $\begin{array}{l}\text { Consciente } \\
\text { Función asociada a } \\
\text { operaciones motrices }\end{array}$ \\
\hline $\begin{array}{l}\text { Operación } \\
\text { asincrónica viable }\end{array}$ & No & $\mathrm{Si}$ & $\mathrm{Si}$ & $\mathrm{Si}$ & No & No & $\mathrm{Si}$ \\
\hline $\begin{array}{l}\text { Referencias teóricas y } \\
\text { estudios de aplicación } \\
\text { de la señal }\end{array}$ & [44] & {$[45][46]$} & {$[47][48]$} & $\begin{array}{l}{[49]} \\
{[50]}\end{array}$ & $\begin{array}{l}{[51]} \\
{[52]}\end{array}$ & $\begin{array}{l}{[53][54]} \\
{[55]}\end{array}$ & [56] \\
\hline
\end{tabular}

Cada día son más las aplicaciones pensadas para los sistemas BCI, actualmente incluyen su uso en personas saludables. Abdulkader, presenta en [59] seis campos diferentes: medicina, ambientes inteligentes y neuroergonómicos, publicidad y neuromarketing, autoregulación educacional, juegos y entretenimientos, y autentificación y seguridad. En esta sección abordaremos las tecnologías de asistencia.

\subsection{Comunicación}

Las investigaciones en esta área están orientadas a reestablecer la comunicación de personas que están impedidas para moverse y hablar, estas incluyen desde un simple selector $\mathrm{Si} / \mathrm{No}$ o $\mathrm{Si} / \mathrm{No} /$ Continuar/Parar, mover un cursor en una pantalla y los deletreadores. Estos últimos se han presentado con diferentes mecanismos de selección; por ejemplo el deletreador basado en P300, fue introducido por Farwel en 1988 [5]; en sus sistemas las 26 letras del alfabeto y algunos otros comandos de control eran desplegados en una pantalla, en una matriz, cuyas frecuencias de parpadeo variaban para las diferentes filas y columnas; permitiendo detectar el carácter seleccionado por el sistema BCI. En sus experimentos se logró que 4 personas sanas comunicaran una palabra de cinco letras a una computadora. Años después Kathner [60], utiliza un deletreador, para probar su sistema P300 con estímulo auditivo y comparará este con el P300 evocado visualmente, la matriz de letras tradicional permite seleccionar el tono objetivo. Nuevamente el sistema fue probado con personas sanas, obteniéndose buenos resultados.

\subsection{Control}

Conforme a las señales del cerebro se establecen un conjunto de comandos que permiten manejar algunos dispositivos sin la utilización de las vías normales: nervios periféricos y músculos. Si bien, la mayoría de las aplicaciones son pensadas para personas imposibilitadas para moverse; también podría servir para facilitar aplicaciones con manos libres o autorregulables de acuerdo con el estado monitoreado de la persona. Dentro de estos sistemas entran los controladores del ambiente, locomoción y neuroprótesis.

\subsection{Controlador Ambiental}

Gao en el 2003 [61] presenta un controlador del ambiente utilizando BCI basado en el potencial de estado estable visualmente evocado (SSVEP). Consta 
de 48 luces verdes, cada uno representando un objetivo, las cuales parpadean a diferentes frecuencias (entre 6 y $15 \mathrm{~Hz}$ ); cuando el operador se fija en una luz determinada, se induce en el cerebro un SSVEP a la misma frecuencia que es identificada por el analizador de frecuencia. Un controlador remoto infrarrojo, previamente entrenado, recibe la selección y controla el aparato seleccionado. Gao logró la selección de canales, el control de volumen y otras funciones de un televisor.

\subsection{Robótica móvil y neuroprótesis}

Nuevamente la mayoría de las investigaciones en esta área, se centran en pacientes que han perdido la movilidad y para los cuales la robótica móvil podría ayudarle a realizar algunas actividades de la vida diaria; sin embargo, las señales del cerebro pueden ser utilizadas, por ejemplo, para controlar la velocidad de un vehículo si se percibe una condición que afecte al conductor.

Dentro de esta línea su uso para lograr un desplazamiento independiente de personas con parálisis, mediante sillas de ruedas basadas en BCI, es el más estudiado. Luzheng divide estos robots móviles en dos categorías: los que son plenamente controlados por el cerebro del usuario o aquellos que combinan las señales BCI con un control inteligente que ayuda al usuario en la navegación [62]. El primer grupo presenta un bajo costo y complejidad computacional, sin embargo, causa al usuario fatiga. El segundo debido a la necesidad de un gran número de sensores tiene un costo mayor y una mayor complejidad computacional. Rebsamen [6] presenta una silla de ruedas del segundo tipo, pero sin un gran número de sensores, por trabajar en un ambiente familiar donde la ruta a seguir por la silla de rueda es establecida con anterioridad. Utiliza un BCI basado en P300 para seleccionar el destino de una lista predefinida que se presenta en una matriz de botones en una pantalla, una vez realizada la selección la silla se moverá en una trayectoria predeterminada; sin embargo, se permite al usuario parar en cualquier momento durante el movimiento, ya sea por un algoritmo rápido P300 o por imágenes motoras. La silla solo requiere dos codificadores rotatorios ópticos utilizados para odometría y un sensor de proximidad para la evasión de obstáculos. Rebsamen solo probó su sistema con personas saludables. Por otro lado, Iñaki propone una silla de ruedas con un sistema de navegación completamente automática [63]. El usuario selecciona un destino inmediato, al enfocar su atención en un lugar de la pantalla, que hace una reproducción visual del escenario, presentándola con un mallado polar; esta selección es captada por el sistema BCI de potencial evocado visualmente P300 y transferida al sistema de navegación autónomo de la silla de ruedas, una vez que el usuario valida la selección. La silla aplica navegación autónoma para moverse hacia la meta mientras evade colisiones al detectar obstáculos por medio de un escáner laser. Nuevamente el prototipo se validó con personas saludables. Carlson [64] también presenta sus estudios con una silla de ruedas con un controlador compartido cerebrocomputadora; pero, en vez de utilizar el paradigma P300, utiliza imágenes motoras que le permiten al usuario interactuar en forma espontánea con la silla hasta alcanzar la posición deseada. Carlson acepta que el tiempo requerido para el entrenamiento es alto, pero lo compara al tiempo de rehabilitación después de un accidente.

\section{Realimentación}

Un problema común que existe entre la actividad BCI de la calibración y entrenamiento (i.e. operación offline) a la actividad en tiempo real (i.e. operación online) es el hecho de que el efecto de la actuación produce perturbaciones que alteran el estado de un sistema y por ende a la modulación de señales EEG en el usuario. A razón de esto, por ejemplo, en casos como la modulación de SMR se han conducido estudios sobre el impacto que tiene la realimentación en la operación BCI [65]. Los mismos han demostrado que mediante diferentes elementos visuales para neurorealimentación en el entrenamiento, se puede influenciar la modulación de la actividad, afectando el sistema BCI [66]. Además, expone que para un óptimo aprendizaje del uso de un sistema BCI, se requieren sistemas visuales de estimulación que afecten en lo menos posible, o en la forma deseada, a los estados mentales de un usuario.

\section{BCI Inteligente}

Otro problema común que ha existido es la no estacionariedad de los datos dentro de un mismo usuario (i.e. cambios en los patrones de las señales en el tiempo). Alternativas conocidas como BCIs 
inteligentes, buscan anticipar la variabilidad en los datos de un usuario, de forma que se prepare una matriz diccionario de esta posible variación y mediante técnicas nuevas de clasificación y filtrado espacial como Spatial Filtering Based Sparsity (SFS) se mejore la operación para BCIs activados mediante M1 (e.g SMR) [67]. Estos métodos, han mejorado la precisión y velocidad de estos sistemas BCI.

\section{Conclusiones}

El entendimiento completo del funcionamiento del cerebro y el diseño de sistemas que puedan asistirlo es todavía un reto, no obstante, a través de la ingeniería actual se han podido implementar soluciones viables a través de aplicación de sistemas BCI. A continuación, las conclusiones más relevantes de la revisión realizada:

- Los sistemas BCI son esencialmente transductores de las señales eléctricas del cerebro, que permiten la interpretación de señales asociadas a procesos mentales.

- En el caso de un método de adquisición de señales no invasivo como el EEG, la captación de las señales de un sistema BCI depende en gran medida de la calidad de los sensores y su ubicación en el cuero cabelludo respecto a una fuente de modulación específica de señal, y la colocación de electrodos debe estar normada de acuerdo al sistema internacional 10-20.

- Todos los dispositivos BCI incluyen una etapa electrónica y una etapa de procesamiento informático que permiten el tratamiento de las señales eléctricas captadas por sensores para lograr una operación de control.

- La operación BCI constituye la aplicación de múltiples disciplinas de las ciencias e ingeniería, como las matemáticas, la electrónica, la teoría de control, la automática y la informática, el desarrollo de cualquiera de estas áreas amplía el campo de acción de los dispositivos BCI.

- El cerebro como tal, está constituido por múltiples regiones que se encargan del procesamiento de información de distinta naturaleza. A razón de esto se derivan señales que son diferentes en todos los aspectos de su modulación. Estas modulaciones, pueden aprovecharse en una forma distinta para constituir unidades de control apropiadas para las interfaces BCI, desde aplicaciones individuales, hasta aproximaciones híbridas.

- La aplicación de técnicas BCI, actualmente tiene relevancia en el campo de la medicina, tanto para el control de dispositivos de asistencias, así como para rehabilitación y/o sustitución de funciones.

\section{Agradecimientos}

Este trabajo es financiado por la Secretaría Nacional de Ciencia, Tecnología e Innovación (SENACYT), bajo contrato $\mathrm{N}^{\circ} 86-2016-4-I D D S 15-094$.

\section{Referencias}

[1] T. C. A. R. Gentiletti G., «Interfaz Cerebro-Computadora: Estado del arte y desarrollo en Argentina,» Revista Argentina de Bioingeniería, vol. 13, n 1, pp. 21-29, 2007.

[2] P. F. Camillo S., «P300-based Brain-computer Interface: clinical applications and new possible directions.,» de Proceedings of SIMPAR 2010 Workshops. Intl. Conf. on Simulation, Modeling and Programming for Autonomous Robots., Germany, 2010.

[3] J. J. Vidal, «Toward direct brain-computer communication.,» Annual review of Biophysics and Bioengineering, vol. 2, $\mathrm{n}^{\circ}$ 1, pp. 157-180, 1973.

[4] R. B. L. W. \&. B. N. Elbert T., «Biofeedback of slow cortical potentials.,» Electroencephalography and Clinical Neurophysiology., vol. 48, n 3, pp. 293-301, 1980.

[5] D. E. Farwell L.A., «Talking off the top of your head: towad a mental prosthesis utilizing event-related brain potentials.,» Electroencephalography and Clinical Neurophysiology., vol. 70, pp. 510-523, 1988.

[6] G. C. Z. H. W. C. T. C. A. J. M. \&. B. E. Rebsamen B., «A brain contolled wheelchair to navigate in familiar environments.,» Neural Systems and Rehabilitation Engineering, IEEE Transactions on, vol. 18, n 6, pp. 590598, 2010.

[7] K. D. W. J. R. Shih J.J., «Brain-computer interfaces in medicine.,» In Mayo Clinic Proceedings. Elsevier, vol. 87, $\mathrm{n}^{\circ} 3$, pp. 268-279, 2012.

[8] C.-N. J. C.-E. R. E.-V. D. Gutiérrez-Martínez J., «Los sitemas de interfaz cerebro-computadora: una herramienta para apoyar la rehabilitación de pacientes con discapacidad motora.,» Mediagraphic.Tecnología en salud., vol. 2, $\mathrm{n}^{\circ} 2$, pp. 62-69, 2013.

[9] I. J. B. L. del Castillo M., «Metodología para la creación de una Interfaz Cerebro.Computador aplicada a la identificación de la Intención del movimiento.,» Revista Iberoamericana de Automática e Informática Industrial., vol. 8, nº 2, pp. 93-102, 2011.

[10] E. W. Jonathan R. Wolpaw, «Brain-Computer Interfaces: 
Something New Under the Sun,» de BCI Principles and Practices, New York, Oxford University Press, 2012, pp. 34.

[11] K. Michio, Future of the Mind, United States: Doubleday, 2014.

[12] F. E. p. 1. C. y. 1. Tecnología., Viaje al universo neuronal., Ministerio de Educación y Ciencia., 2007.

[13] K. Academy, «La sinapsis,» [En línea]. Available: https://es.khanacademy.org/science/biology/humanbiology/neuron-nervous-system/a/the-synapse. [Último acceso: 20 enero 2017].

[14] D. N. Allan R. Crossman, «Componentes y organización del sistema nervioso: Neuronas y neuroglía,» de Neuroanatomía: Texto y Atlas 5ta Edición, Barcelona, Elsevier, 2015, pp. 2-3.

[15] K. Academy., «Función y estructura de la neurona.,» [En línea]. Available: https://es.khanacademy.org/science/biology/humanbiology/neuron-nervous-system/a/overview-of-neuronstructure-and-function. [Último acceso: 20 enero 2017].

[16] E. D. A. f. t. Brain, Neuroscience: Science of the Brain-An Introduction for Young Students., The British Neuroscience Association, 2003.

[17] P. Ceibal, «El sistema nervioso humano. La corteza cerebral,» [En línea]. Available: http://www.ceibal.edu.uy/userfiles/P0001/ObjetoAprendiza je/HTML/Unidad_elsistemanervisohumano_AWilliman.elp /la_corteza_cerebral.html. [Último acceso: 16 enero 2017].

[18] G. R., «Inferior olivary neurons in the awake cat: Detection of contact and passive body displacement,» $\mathrm{J}$. Neurophysiol, ${ }^{\circ}$ 54, pp. 40-60, 1985.

[19] L. S. Stone LS, «Detection of tracking errors by visual climbing fiber inputs to monkey cerebellar flocculus during pursuit eye movements.,» Neurosci Lett, $\mathrm{n}^{\circ} 72$, pp. 163$168,1986$.

[20] W. J. E. T. Kim JH, «Climbing fiber afferent modulation during treadmill locomotion in the cat,» J. Neurophysiol, $\mathrm{n}^{\circ}$ 57, pp. 787-802, 1987.

[21] K. J. E. T. Wang JJ, «Climbing fiber afferent modulation during a visually guided, multi-joint arm movement in the monkey,» Brain Res, n 410, pp. 323-329, 1987.

[22] B. J. Lou JS, «Responses of sagittally aligned Purkinje cells during perturbed locomotion: Synchronous activation of climbing fiber inputs,» J. Neurophysiol, nº 68, pp. 570$580,1992$.

[23] U. D. o. H. a. H. S. N. I. o. Health., «Medline Plus. Información de salud para usted.,» [En línea]. Available: https://medlineplus.gov/spanish/ency/esp_imagepages/1924 4.htm. [Último acceso: 16 enero 2017].

[24] M. Clinic, «Mayfield Brain\&Spine,» [En línea]. Available: http://www.mayfieldclinic.com/PDF/PE-AnatBrain.pdf.
[Último acceso: 20 enero 2017].

[25] J. Sánchez, «Técnicas de toma de datos y análisis de Electroencefalografía.,» Universidad de Sevilla, Sevilla, 2014.

[26] L. Palacios, «Breve historia de la electroencefalografía,» Acta Neurol Colomb., vol. 18, n² 2, pp. 104-107, 2002.

[27] E. Fine, «Berger Hans de Encyclopedia of the Neurological Sciences.,» Second Editon of Academic Press Oxford, 2014, p. 411.

[28] R. Navarro, «Instrumentación Biomédica Electroencefalografía. Departamento de Electrónica.,» Universidad de Alcalá, Alcalá, 1998.

[29] L. Palacios, «Breve Historia de la Electroencefalografía,» Acta Neurol Colomb, vol. 18, nº 2, pp. 104-107, 2002.

[30] E. Val, Tesis Doctoral: Utilidad de la Cartografía cerebral en el diagnóstico del TDAH., Burgos: Universidad de Burgos., 2014.

[31] R. Navarro, Instrumentación Biomédica: Electroencefalografía, Alcalá: Universidad de Alcalá, 1998.

[32] P. L. Nunez, «Electric and Magnetic Fields Produced by the Brain: Macroscale fields,» de BCI Principles and Practices, New York, Oxford University Press, 2012, p. 45.

[33] J. Sánchez, Técnicas de toma de datos y análisis de Electroencefalografía, Sevilla: Universidad de Sevilla, 2014.

[34] P. L. Nunez, «All Scalp Recordings are Bipolar,» de BCI Principles and Practices, New York, Oxford University Press, 2012, p. 57.

[35] D. J. M. J. C. P. Dean J. Krusienski, «BCI Signal Processing: Feature Extraction,» de BCI Principles and Practices, New York, Oxford University Press, 2012, pp. 123-144.

[36] H. Wang, Y. Li y L. \&. Y. G. Long, «An asynchronous wheelchair control by hybrid EEG-EOG,» Congnitive Neurodynamics, vol. 8, pp. 399-409, 2014.

[37] K. D. S. W. W. J. McFarland DJ., «Emulation of computer mouse control with a noninvasive brain computer interface.,» Journal Neural Engineering., vol. 5, $\mathrm{n}^{\circ}$ 2, pp. 101-110, 2008.

[38] H. B., «An Online Transformation of EEG scalp potentials into ortogonal source derivations,» Electroencephalography Clin Neurophysiol, $n^{\circ} 39$, pp. 526-530.

[39] R. P. Rao, Brain-Computer Interfacing, New York: Cambridge University Press, 2013, pp. 8-9.

[40] D. J. \&. K. D. J. McFarland, «BCI Signal Processing: Feature Translate,» de BCI principles and practices, Oxford University Press, 2012, pp. 147-163.

[41] N. Birbaumer, T. Hinterberger y A. \&. N. N. Kübler, «The thought-translation device: Neurobehavioral mechanisms and clinical outcomes.,» IEEE Transactions on Neural Systems and Rehabilitation Engineering., vol. 11, pp. 120- 
123, 2003.

[42] B. Blankertz, G. Dornege, C. Schafer, R. Krepki, J. Kohlnorgen, M. K-R., V. Kunzamann y F. \&. C. G. Losch, «Boosting bit rates and error detection for the classification of fast-paced motor commands based on single trial EEG analysis.,» IEEE Transactions on Rehabilitation Engineering, vol. 11, pp. 100-104, 2003.

[43] J. Wolpaw, N. Birbaumer, D. McFarland y G. \&. V. T. Pfurtscheller, «Brain-computer interfaces for communication and control,»Clinical Neurophysiology, vol. 113, pp. 767-791, 2002.

[44] T. W. Picton, «The P300 Wave of the Human EventRelated Potential,» J. Neurophysiology, vol. 4, n $\mathrm{n}^{\mathrm{o}}$ 9, pp. 456-479, 1992.

[45] P. N. S. Celesia G., «Visual evoked potentials and electroretinograms.,» de Electroencephalography - Basic Principles, clinical applications and related fields., Philadelphia, Lippincott Williams \& Wilkins, 2005, pp. 1017-1043.

[46] G. X. W. Y. H. B. Bin G., «VEP-based brain-computer interfaces: time, frequency, and code modulations.,» Computational Intelligence Magazine, IEEE, $n^{\circ} 4$, pp. 2226, 2009.

[47] R. S. C. N. G. P. Müller-Putz, «Steady-State Somatosensory Evoked Potentials: Suitable Brain Signals for Brain-Computer Interfaces?,» IEEE Transactions on Neural Systems and Rehabilitation Engineering, vol. 14, $\mathrm{n}^{\circ}$ 1, pp. 30-37, 2006.

[48] K. K. S. C. J. Sangtae Ahn, «Steady-State Somatosensory Evoked Potential for Brain-Computer Interface-Present and Future,» Frontiers in Human Neuroscience, 14 Enero 2016. [En línea]. Available: https://www.ncbi.nlm.nih.gov/pmc/articles/PMC4712271/.

[49] D. L. J. B. S. G. H. T. W. P. David R. Stapelis, «Huma Auditory Steady State Potentials,» Electrophysiologic Techniques in Audiology and Otology, vol. 5, $\mathrm{n}^{\mathbf{o}}$ 2, pp. 105-113, 1984.

[50] P. d. L. M. I. d. A. S. K. C. L. d. A. O. G. L. Aline Tenório Lins Carnaúba, «SciELO-Auditory Steady-State Evoked Potentials at Carrier Frequencies abore $4000 \mathrm{~Hz}$,» Audiology - Communication Research, 03 Julio 2017. [En línea]. Available: www.scielo.br/scielo.php?pid=S231764312017000100320\&script=sci_arttext\&tlng=en.

[51] «Bereitschaftspotentials. Movement-Related Cortical Potentials,» Journal of Psychophysiology, vol. 3, n 17, pp. 135-136, 2013.

[52] N. B. A. R.-M. Ozge Yilmaz, «NCBI - Movement related slow cortical potentials in severely paralyzed chronic stroke patients,» Frontiers in Human Neuroscience, 15 Enero 2015. [En línea]. Available: https://www.ncbi.nlm.nih.gov/pmc/articles/PMC4295525/.

[53] Altenmüller, «Neurocognitive functions and the EEG.,» de
Electroencephalography: basic principles, clinical applications and related fields, Philadelphia, Lippincott Williams \& Wilkins, 2005, pp. 661 - 682.

[54] W. W. G., «Contingent Negative Variation: an electric sign of sensorimotor association and expentancy in the human brain,» Nature, $n^{\circ}$ 203, pp. 380-384, 1964.

[55] B. A. H. L. T. A. W. I. D. R. S. G. M. H. H. A. R. Holger Gevensleben, «NCBI - Neurofeedback of slow cortical potentials: neural mechanisms and feasibility of a placebocontrolled disegn in healty adults,» Frontiers in Human Neuroscience, 11 Diciembre 2014. [En línea]. Available: https://www.ncbi.nlm.nih.gov/pmc/articles/PMC4263073/.

[56] D. J. McFarland, L. A. Miner y T. M. \&. W. J. R. Vaughan, «Mu and Beta Rhythm Topographies During Motor Imagery and Actual Movements,» Brain Topography, vol. 12, n 3, pp. 177-186, 2000.

[57] W. J. Mark J., «Clinical Applications of Brain-Computer Interfaces: Current State and future Prospects.,» IEEE Reviews in Biomedical Engineering., vol. 2, pp. 187-199, 1999.

[58] M. Moore., «Real world applications for Brain-computer Interface Technology.,» IEEE Transactions on Neural Systems and Rehabilitation Engineering., vol. 11, $\mathrm{n}^{\mathrm{o}} 2$, pp. 162-165, 2003.

[59] A. A. M. M. Abdulkader S., «Brain Computer Interfacing: Applications and Challenges.,» Egyptian Informatics Journal., vol. 16, pp. 213-230, 2015.

[60] R. C. P. E. B. C. B. N. H. S. Kathner I., «A portable auditory $\mathrm{P} 300$ brain-computer interface with directional cues.,» Clinical Neurophysiology., vol. 124, no 2, pp. 327338, 2013.

[61] X. D. C. M. G. S. Gao X., «A BCI-based environmental controller for the motion-disabled., IEEE Transactions Neural Systems Rehabilitation Engineering., vol. 11, $\mathrm{n}^{\mathrm{o}} 2$, pp. 137-140, 2003.

[62] F. X. L. Y. Bi L., «EEG-based brain controlled mobile robots: a survey,» IEEE Transactions on Human-Machine Systems, vol. 43, n 2, pp. 161-176, 2013.

[63] A. J. K. A. Iturrate I., «A Noninvasive Brain-Actuated Wheelchair Based on a P300 Neurophysiological Protocol and Automated Navigation.,» IEEE Transactions on Robotics., vol. 25, n 3, pp. 614-627, 2009.

[64] M. J. Carlson T., «Brain-controlled wheelchairs,» IEEE Robotics\&Automation Magazine, pp. 65-73, 2013.

[65] M. Z. Y. A. M. n. S. K. D. M. Eltaf Abdalsalam, «Modulation of sensorimotor rhythms for brain-computer interface using motor imagery with online feedback,» Springer-Verlag, $\mathrm{n}^{\circ}$ 12, pp. 557-564, 2017.

[66] P. F. E. Z. Dariusz Zapala, «The Impact of Differente Visual Feedbacks in User Training on Motor Imagery Control in BCI,» Appl Psychophysiol Biofeedback, $n^{\circ} 43$, pp. 23-35, 2017. 
[67] J. R. D. S. P. M. M. S. S. R. Sreeja, «Classification of Motor Imagery Based EEG Signals Using Sparsity Approach,» Intelligent Human Computer Interaction Proceedings, $n^{\circ}$ 9, pp. 47-59, 2017.

[68] OCDE, La comprensión del cerebro. El nacimiento de una ciencia del aprendizaje., Ediciones Universidad Católica Silva Henríquez, 2009.

[69] L. d. S. G. Pfurtscheller, «EEG. event-related desynchronization (ERD) and event-related synchronization ERS),» de Niedermeyer's Electroencephalography 6th Edition, Philadelphia, Wolters Kluwer, 2011, pp. 935-948. 